Discussion Paper No. 825

\title{
WEITZMAN MEETS NORDHAUS: \\ EXPECTED UTILITY AND \\ CATASTROPHIC RISK \\ IN A STOCHASTIC, \\ ECONOMY-CLIMATE MODEL
}

\author{
Masako Ikefuji \\ Roger J. A. Laeven \\ Jan R. Magnus \\ Chris Muris
}

December 2011

The Institute of Social and Economic Research

Osaka University

6-1 Mihogaoka, Ibaraki, Osaka 567-0047, Japan 


\title{
Weitzman meets Nordhaus: Expected utility and catastrophic risk in a stochastic economy-climate model*
}

\author{
December 15, 2011 \\ Masako Ikefuji \\ Institute of Social and Economic Research, Osaka University \\ Roger J. A. Laeven \\ Department of Econometrics $\&$ Operations Research, Tilburg University \\ Jan R. Magnus \\ Department of Econometrics 83 Operations Research, Tilburg University \\ Chris Muris \\ Department of Economics, Simon Fraser University
}

\footnotetext{
*We are very grateful to Graciela Chichilnisky, John Einmahl, Reyer Gerlagh, Johan Eyckmans, Sjak Smulders, Peter Wakker, Aart de Zeeuw, Amos Zemel, and seminar and conference participants at Kyushu University, Tilburg University, EAERE 2011 in Rome, and the Tilburg Sustainability Center for helpful comments. This research was funded in part by the JSPS under grant C-22530177 (Ikefuji) and by the NWO under grant Vidi-2009 (Laeven). E-mail addresses: Ikefuji@iser.osaka-u.ac.jp (Ikefuji), R.J.A.Laeven@uvt.nl (Laeven), Magnus@uvt.nl (Magnus), CMuris@sfu.ca (Muris).
} 


\title{
Corresponding author:
}

Roger J. A. Laeven

Department of Econometrics and Operations Research

Tilburg University

P.O. Box 90153

5000 LE Tilburg

The Netherlands

e-mail: R.J.A.Laeven@uvt.nl

\begin{abstract}
We specify a stochastic economy-climate model, adapting Nordhaus' deterministic economy-climate model by allowing for Weitzman-type stochasticity. We show that, under expected power utility, the model is fragile to heavy-tailed distributional assumptions and we derive necessary and sufficient conditions on the utility function to avoid fragility. We solve our stochastic economy-climate model for two cases with compatible pairs of utility functions and heavy-tailed distributional assumptions. We further develop and implement a procedure to learn the input parameters of our model and show that the model thus specified produces robust optimal policies. The numerical results indicate that higher levels of uncertainty lead to less abatement and consumption, and to more investment.
\end{abstract}

JEL Classification: D61; D81; Q5.

Keywords: Economy-climate models; Catastrophe; Expected utility; Heavy tails; Power utility. 


\section{Introduction}

An economist, when asked to model decision making under risk or uncertainty for normative purposes, would typically work within the expected utility framework with constant relative risk aversion (that is, power utility). A statistician, on the other hand, would model economic catastrophes through probability distributions with heavy tails. Unfortunately, expected power utility is fragile with respect to heavy-tailed distributional assumptions: expected utility may fail to exist or it may imply conclusions that are 'incredible'.

Economists have long been aware of this tension between the expected utility paradigm and distributional assumptions (Menger, 1934), and the discussions in Arrow (1974), Ryan (1974), and Fishburn (1976) deal explicitly with the trade-off between the richness of the class of utility functions and the generality of the permitted distributional assumptions. Compelling examples in Geweke (2001) corroborate the fragility of the existence of expected power utility with respect to minor changes in distributional assumptions.

The combination of heavy-tailed distributions and the power utility family may not only imply infinite expected utility, but also infinite expected marginal utility, and hence, via the intertemporal marginal rate of substitution (the pricing kernel), lead to unacceptable conclusions in cost-benefit analyses. For example, with heavy-tailed log-consumption and power utility, the representative agent should postpone any unit of current consumption to mitigate future catastrophes. The latter aspect was recently emphasized by Weitzman (2009) in the context of catastrophic climate change. Weitzman also argues that attempts to avoid this unacceptable conclusion will necessarily be non-robust.

In this paper we study the question of how to conduct expected utility analysis in the presence of catastrophic risks, in the context of extreme climate change. Our paper is built on four beliefs, which will recur in our analysis:

Catastrophic risks are important. To study risks that can lead to catastrophe is important in many areas, e.g., financial distress, traffic accidents, dike bursts, killer asteroids, nuclear power plant disasters, and extreme climate change. Such low-probability high-impact events should not be ignored in cost-benefit analyses for policy making.

A good model 'in the center' is not necessarily good 'at the edges'. Models are approximations, not truths, and approximations may not work well if we 
move too far away from the point of approximation. In our context, the widely adopted family of power utility functions, often appropriate when one considers large inputs remote from zero, may not work well for decision making under heavy-tailed risks with non-negligible support beyond the usual domain of inputs.

The price to reduce catastrophic risk is finite. Are we willing to spend everything to avoid children being killed at a dangerous street? Or dikes to burst? Or a power plant to explode? Or a killer asteroid to hit the Earth? Or climate to change rapidly? No, we are not. To assume the opposite (that a society would be willing to offer all of its current wealth to avoid or mitigate catastrophic risks) is not credible, not even from a normative perspective. In our context, there is a limit to the amount of current consumption that the representative agent is willing to give up in order to obtain one additional certain unit of future consumption, no matter how extreme and irreversible climate change may be. In other words: the expected pricing kernel is finite.

Light-tailed risks may result in heavy-tailed risk. When $x$ is normally distributed (light tails) then $1 / x$ has no moments (heavy tails). Also, when $x$ is normally distributed then $e^{x}$ has finite moments, but when $x$ follows a Student distribution then $e^{x}$ has no moments. In the context of extreme climate change: temperature has fluctuations but one would not expect heavy tails in its distribution. This does not, however, imply that functions of temperature cannot have heavy tails. For example, it may well be reasonable to use heavy-tailed distributional assumptions to model future (log) consumption.

There is an important literature on stochastic economy-climate models (see, for example, Keller et al., 2004, Mastrandrea and Schneider, 2004, and the references therein). The integrated assessment models of climate economics are, however, predominantly deterministic and rarely incorporate catastrophic risk (Ackerman et al., 2010). To allow for uncertainty and extreme climate change, we start by specifying a stochastic economy-climate model, adapting Nordhaus' (2008) deterministic dynamic integrated climate and economy (DICE) model by allowing for stochasticity in the spirit of Weitzman (2009). We show formally that, under expected power utility, the model is indeed fragile to heavy-tailed distributional assumptions, and we derive necessary and sufficient conditions on the utility function to avoid fragility, thus ensuring that expected utility and expected marginal utility (hence the expected pricing kernel) are finite, also under heavy-tailed distributional assumptions.

Next, we solve a two-period version of the model, first with power utility and light-tailed distributional assumptions. Since the assumption of expected power utility is incompatible with heavy-tailed distributional assumptions, we then restrict attention to utility functions that satisfy the derived compat- 
ibility conditions, and solve our stochastic economy-climate model with the well-known exponential utility function and also with the less well-known (but more suitable) 'Pareto utility' function, under both light-tailed and heavy-tailed distributional assumptions.

Completing the resulting model requires specifying a number of model parameters as inputs. These parameters cannot 'simply' be determined by conventional statistical inference based on historical data. We discuss how to set the model parameters in a process towards agreement, using experts' priors on parameter values, and learning about parameters from resulting optimal model output. The key to this learning and agreement process is the translation of model parameters that are relatively difficult to interpret into quantities that allow a more straightforward interpretation. Contrary to Weitzman's (2009) claim, we find that our optimal policies thus derived are quite robust with respect to minor and reasonable changes to the input parameters. This means that the policymaker is not left empty-handed when it comes to cost-benefit analyses under catastrophic risk.

Our numerical analysis indicates that allowing for heavy-tailed distributional assumptions in extreme climate change modeling, inducing both heavy-tailed downside risk and heavy-tailed upward potential, leads to a reduction of current abatement and consumption and to an increase in current investment, when compared to a deterministic analysis. The increase in current investment may be interpreted via precautionary savings. While the differences are clearly visible, they are not unlimited.

The paper is organized as follows. In Section 2 we propose a simplified version of Nordhaus' economy-climate model. In Section 3 we introduce uncertainty in the spirit of Weitzman. This is the first new feature added to the Nordhaus model. Section 4 discusses expected (marginal) utility and uncertainty in a more general setting, deriving results on the trade-off between permitted distributional assumptions and the existence of expected (marginal) utility. In Section 5 we specialize the model to two periods only, and add a second new feature to the Nordhaus model: scrap value functions. In Section 6, we present (partial) results for power utility, which is incompatible with heavy tails, and for exponential and 'Pareto' utility, which are compatible with heavy tails. Section 7 discusses how to learn the parameters of our model and calibrate policy using information such as the probability of catastrophe, and reports on robustness tests. Section 8 concludes. There are two appendices: Appendix A provides the Kuhn-Tucker conditions and Appendix B contains proofs of the propositions. 


\section{Nordhaus simplified}

Our framework is a simple economy-climate model in the spirit of Nordhaus and Yang (1996) and Nordhaus (2008). Everybody works. In period $t$, the labor force $L_{t}$ together with the capital stock $K_{t}$ generate GDP $Y_{t}$ through a Cobb-Douglas production function

$$
Y_{t}=A_{t} K_{t}^{\gamma} L_{t}^{1-\gamma} \quad(0<\gamma<1)
$$

where $A_{t}$ represents technological efficiency and $\gamma$ is the elasticity of capital. Capital is accumulated through

$$
K_{t+1}=(1-\delta) K_{t}+I_{t} \quad(0<\delta<1),
$$

where $I_{t}$ denotes investment and $\delta$ is the depreciation rate of capital. Production generates carbon dioxide (CO2) emissions $E_{t}$ :

$$
E_{t}=\sigma_{t}\left(1-\mu_{t}\right) Y_{t}
$$

where $\sigma_{t}$ denotes the emissions-to-output ratio for $\mathrm{CO} 2$, and $\mu_{t}$ is the abatement fraction for $\mathrm{CO} 2$. The associated $\mathrm{CO} 2$ concentration $M_{t}$ accumulates through

$$
M_{t+1}=(1-\phi) M_{t}+E_{t} \quad(0<\phi<1),
$$

where $\phi$ is the depreciation rate of $\mathrm{CO} 2$ (rate of removal from the atmosphere). Temperature $H_{t}$ develops according to

$$
H_{t+1}=\eta_{0}+\eta_{1} H_{t}+\eta_{2} \log \left(M_{t+1}\right) \quad\left(\eta_{1}>0, \eta_{2}>0\right) .
$$

In each period $t$, the fraction of GDP not spent on abatement or 'damage' is either consumed $\left(C_{t}\right)$ or invested $\left(I_{t}\right)$ along the budget constraint

$$
\left(1-\omega_{t}\right) d_{t} Y_{t}=C_{t}+I_{t}
$$

The temperature-impact function $d_{t}$ depends only on temperature and satisfies $0<d_{t} \leq \bar{d}_{t}$, where $\bar{d}_{t}$ represents the optimal temperature for the economy. Deviations from the optimal temperature cause damage. We specify $d_{t}$ as

$$
d_{t}=\frac{\bar{d}_{t}}{1+\xi H_{t}^{2}} \quad(\xi>0)
$$

For very high and very low temperatures $d_{t}$ approaches zero. The optimal value of $d_{t}$ occurs at $H_{t}=0$ (the temperature in 1900, as in Nordhaus) when $d_{t}=\bar{d}_{t}$. Hence, 'net' output $d_{t} Y_{t}$ is a fraction, not of $Y_{t}$ as in Nordhaus, but 
of $\bar{d}_{t} Y_{t}$, the output achievable under optimal climate conditions. A fraction $\omega_{t}$ of $d_{t} Y_{t}$ is spent on abatement, and we specify the abatement cost fraction as

$$
\omega_{t}=\psi_{t} \mu_{t}^{\theta} \quad(\theta>1) .
$$

If $\mu_{t}$ increases then so does $\omega_{t}$, and a larger fraction of GDP will be spent on abatement. These equations capture the essence of the Nordhaus (2008) DICE model.

The model includes stock variables $L_{t}, K_{t}, M_{t}$, and $H_{t}$, fractions $\omega_{t}$ and $\mu_{t}$, and scale variables $A_{t}, d_{t}, \sigma_{t}$, and $\psi_{t}$, all measured at the beginning of period $t$; and flow variables $Y_{t}, C_{t}, I_{t}$, and $E_{t}$, all measured in period $t$ (not in year $t$ ). Notice that $L_{t}$ is a stock, not a flow. As in Nordhaus (2008) one period is ten years. We choose the exogenous variables such that $L_{t}>0$, $A_{t}>0, \sigma_{t}>0$, and $0<\psi_{t}<1$. The policy variables must satisfy

$$
C_{t} \geq 0, \quad I_{t} \geq 0, \quad 0 \leq \mu_{t} \leq 1
$$

With these restrictions all variables will have the correct signs and all fractions will lie between zero and one.

Given a utility function $U$ we define welfare in period $t$ as

$$
W_{t}=L_{t} U\left(C_{t} / L_{t}\right)
$$

If the policy maker has an infinite horizon, then he/she will maximize total discounted welfare,

$$
W=\sum_{t=0}^{\infty} \frac{W_{t}}{(1+\rho)^{t}} \quad(0<\rho<1)
$$

where $\rho$ denotes the discount rate. Letting $x$ denote per capita consumption, the utility function $U(x)$ is assumed to be defined and strictly concave for all $x>0$. There are many such functions, but a popular choice is

$$
U(x)=\frac{x^{1-\alpha}-1}{1-\alpha} \quad(\alpha>0)
$$

where $\alpha$ denotes the elasticity of marginal utility of consumption. This is the so-called power function. Many authors, including Nordhaus (2008), select this function and choose $\alpha=2$ in which case $U(x)=1-1 / x$. Also popular is $\alpha=1$; see Kelly and Kolstad (1999) and Stern (2007).

Our interest is in maximizing welfare $W$ with respect to the policy bundles $\left(C_{t}, I_{t}, \mu_{t}\right)$ for $t=0,1,2, \ldots$ We choose the exogenous variables $L_{t}, A_{t}, \sigma_{t}$, and $\psi_{t}$ as in Nordhaus (2008), and we let $\bar{d}_{t} \equiv 1$ and $\alpha=2$. Calibrating the 
Table 1: Comparison of stocks in Nordhaus (DICE) and our (SICE) models

\begin{tabular}{lrrrrrrrr}
\hline & \multicolumn{2}{c}{2005} & \multicolumn{2}{c}{2055} & \multicolumn{2}{c}{2105} & \multicolumn{2}{c}{2155} \\
& DICE & SICE & DICE & SICE & DICE & SICE & DICE & SICE \\
\hline$K$ & 137 & 137 & 353 & 354 & 707 & 711 & 1317 & 1324 \\
$M$ & 809 & 809 & 1048 & 988 & 1270 & 1233 & 1428 & 1430 \\
$H$ & 0.7 & 0.7 & 1.8 & 1.5 & 2.7 & 2.4 & 3.3 & 3.2 \\
\hline
\end{tabular}

parameters and initial values (presented in our background document, see Ikefuji et al., 2011b), our GAMS code ${ }^{1}$ produces optimal values over sixty periods that are very close to the values obtained in Nordhaus, as shown in Table 1. Hence it appears that our simplified version of the DICE model (hereafter, SICE = simplified DICE) works as well as the original version.

\section{$3 \quad$ Enter Weitzman and uncertainty}

Weitzman (2009) recently noted, in a highly stylized setting, that heavytailed uncertainty and power utility are incompatible, since this combination of uncertainty and preferences implies an infinite expected pricing kernel. In order to avoid this, Weitzman introduces a lower bound on consumption. He then argues that this lower bound is related to a parameter that resembles the value of a statistical life, and proves that the expected pricing kernel approaches infinity as the value of this parameter approaches infinity (the 'dismal theorem'). Weitzman further argues that this 'VSL-like' parameter is hard to know, and interprets this result as follows:

"... reasonable attempts to constrict the length or the heaviness of the 'bad' tail (or to modify the utility function) still can leave us with uncomfortably big numbers whose exact value depends non-robustly upon artificial constraints or parameters that we really do not understand." (Weitzman, 2009, p. 11)

We agree with Weitzman that incompatible pairs of utility functions and distribution functions exist, in the sense that the expected pricing kernel or other important policy variables become infinite. In fact, we derive in Section 4 necessary and sufficient conditions on the utility functions for the expected pricing kernel to exist, also under heavy tails. But we object to the dismal theorem for two reasons. As we demonstrate formally in Section 4 and numerically in Section 6, the dismal theorem is based on an incompatible (invalid) model specification; it is avoided when the economic model

\footnotetext{
${ }^{1}$ Available at http://www.janmagnus.nl/items/risk.pdf
} 
(utility function) is compatible with the statistical model (heavy tails). Furthermore, as we show in Section 7, more effort can be made to know an input parameter that is 'hard to know', and we develop and implement a learning-and-agreement procedure for precisely this purpose.

We introduce uncertainty in the simplified Nordhaus model in a Weitzmanlike manner. There is much uncertainty in the economics of climate change (Manne and Richels, 1992; Nordhaus, 1994; Weitzman, 2009). There is model uncertainty, parameter uncertainty, and uncertainty about the possible reduction of parametric variability over time (updating); see Kelly and Kolstad (1999) and Leach (2007). We model uncertainty through stochasticity. In the literature, stochasticity is typically introduced through the damage function (Roughgarden and Schneider, 1999; Mastrandrea and Schneider, 2004) or through a random shock in temperature (Kelly and Kolstad, 1999; Leach, 2007). We follow this literature by introducing stochasticity through the temperature-impact function $d_{t}$, more precisely through $\bar{d}_{t}$, the impact under optimal temperature. We are uncertain about the optimal temperature, because we are uncertain about the correctness of the functional form of $d_{t}$, about the values of the parameters, and about the underlying temperature equation. We capture these three sources of uncertainty by writing

$$
\bar{d}_{t}=e^{-\tau^{2} / 2} e^{\tau \epsilon_{t}},
$$

where $\epsilon_{t}$ denotes a random error with mean zero and variance one. This implies that 'net GDP' is given by

$$
d_{t} Y_{t}=\frac{e^{-\tau^{2} / 2} Z_{t}}{1+\xi H_{t}^{2}}, \quad Z_{t}=A_{t} K_{t}^{\gamma} L_{t}^{1-\gamma} e^{\tau \epsilon_{t}},
$$

so that random noise enters the Cobb-Douglas production function in the usual 'linear' way when we write $\log \left(Z_{t} / L_{t}\right)=\log A_{t}+\gamma \log \left(K_{t} / L_{t}\right)+\tau \epsilon_{t}$.

Introducing uncertainty in this way is in the spirit of Weitzman and it is convenient for our purpose. An alternative, possibly more intuitive, specification is to let stochasticity enter through a random multiplicative shock in temperature, by setting $\bar{d}_{t}=1$ and randomizing the parameter $\xi$. This leads to an alternative specification of the temperature-impact function,

$$
d_{t}^{\text {alt }}=\left[1+u \xi H_{t}^{2}\right]^{-1}, \quad u=e^{\tau^{2} / 2} e^{-\tau \epsilon_{t}},
$$

as opposed to our original specification $d_{t}=\left[u\left(1+\xi H_{t}^{2}\right)\right]^{-1}$. For our analysis, large values of $u$ are relevant. For large $u$ we have approximately $d_{t}^{\text {alt }} \approx$ $\left[u \xi H_{t}^{2}\right]^{-1}$, whose behavior is similar to $d_{t}$. Hence, for our purpose the two specifications are conceptually equal. 
If $\epsilon_{t}$ follows a normal distribution $\mathrm{N}(0,1)$, then the moments of $\bar{d}_{t}$ exist, and we have $\mathrm{E}\left(\bar{d}_{t}\right)=1$ and $\operatorname{var}\left(\bar{d}_{t}\right)=e^{\tau^{2}}-1$. Since the distribution of $\bar{d}_{t}$ is heavily skewed, more uncertainty (higher $\tau$ ) implies more probability mass of $\bar{d}_{t}$ close to zero, and a higher probability of damage. If, however, we move only one step away from the normal distribution and assume that $\epsilon_{t}$ follows a Student distribution with any (finite) degrees of freedom, then the expectation is infinite (Geweke, 2001). This fact predicts that expected welfare may be very sensitive to distributional assumptions: random noise with finite moments (Student distribution) may turn into random variables without moments $\left(\bar{d}_{t}, d_{t} Y_{t}\right)$.

We need sensible values for the uncertainty parameter $\tau$. The stochasticity, as given in (3), captures uncertainty about GDP that is due to uncertainty about climate change. Historical variation in GDP may therefore serve as an initial upper bound proxy for $\tau$. Barro (2009) calibrates the standard deviation of $\log$ GDP to a value of 0.02 on an annual basis. Over a 10year horizon this would correspond to about 0.06, under normality. Barro, however, only considers rich (OECD) countries, which means that for our purposes this value needs to be scaled up. In addition to the value of $\tau$ we need to consider the question whether or not the uncertainty introduced is indeed heavy-tailed. A (partial) answer to this question is contained in a recent paper by Ursúa (2010) who claims that the growth rate of GDP indeed features heavy tails.

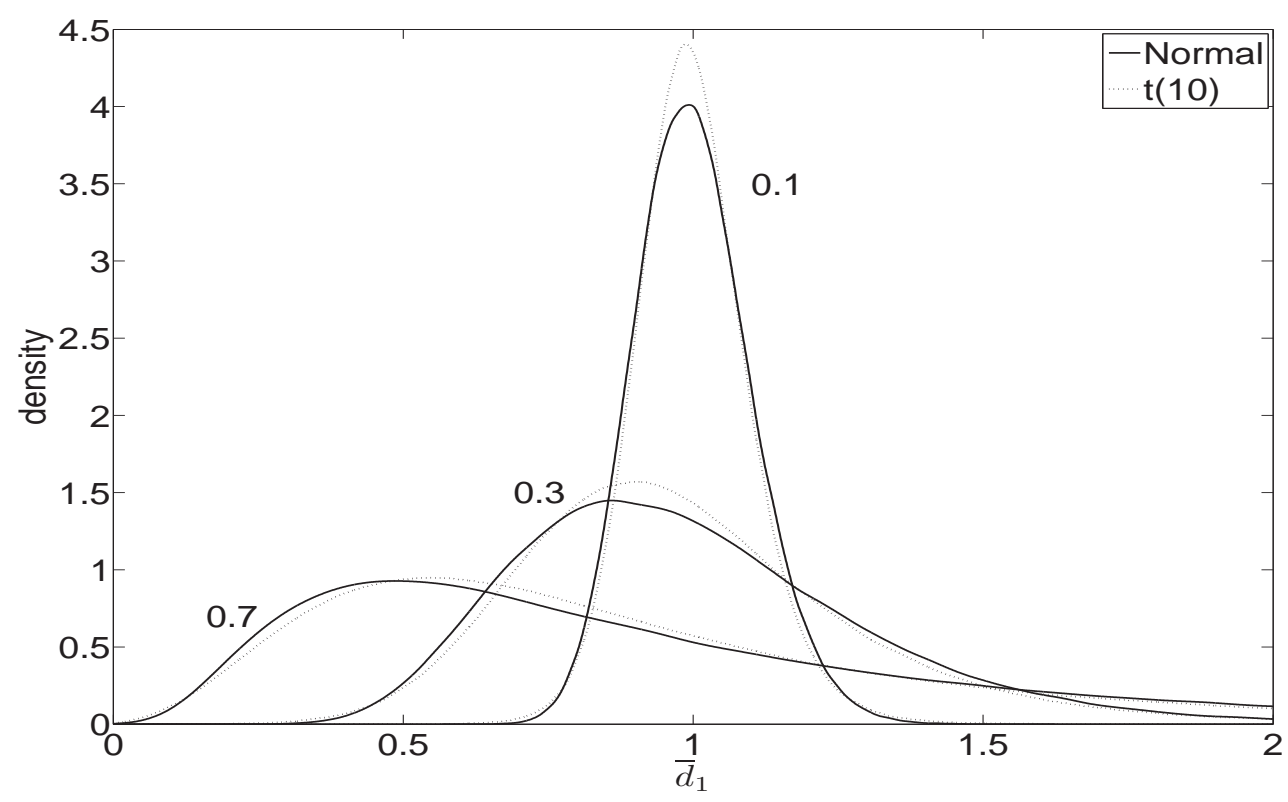

Figure 1: Density of $\bar{d}_{t}$ for $\tau=0.1,0.3$, and 0.7 
In Figure 1 we plot the density of $\bar{d}_{t}$ for three values of $\tau: 0.1,0.3$, and 0.7 , both when $\epsilon_{t}$ follows a $\mathrm{N}(0,1)$ distribution (solid line) and when $\epsilon_{t}=\sqrt{4 / 5} u$, where $u$ follows a Student distribution (as adopted in Weitzman, 2009) with 10 degrees of freedom (which implies a 'tail index' that is broadly consistent with the empirical analysis in Ursúa, 2010). Notice that $\mathrm{E}\left(\epsilon_{t}\right)=0$ and $\operatorname{var}\left(\epsilon_{t}\right)=1$ in both cases. When $\tau=0.1$, we see that almost $100 \%$ of the distribution of $\bar{d}_{t}$ lies in the interval $(0.5,2.0)$, both for the $\mathrm{N}(0,1)$ distribution and for the $t(10)$ distribution. When $\tau=0.3,97.8 \%$ (97.2\% for the Student distribution) lies in the interval $(0.5,2.0)$; and, when $\tau=0.7$, only $64.9 \%$ (67.2\% for the Student distribution) lies in this interval. We conclude that $\tau=0.7$ may serve as a credible upper bound for the uncertainty range, and hence we report our results for $\tau=0.0,0.3,0.5$, and 0.7 .

\section{$4 \quad$ Expected utility and heavy tails}

Since the axiomatization of expected utility (EU) by Von Neumann and Morgenstern (1944) and Savage (1954), numerous objections have been raised against it. Most of these relate to empirical evidence that the behavior of agents under risk and uncertainty does not agree with EU. Despite important developments in non-expected utility theory, EU remains the dominant normative decision theory (Broome, 1991; Sims, 2001), and the current paper stays within the framework of EU. Our results presented below corroborate the fact that expected utility theory may reliably provide normatively appealing results, also in the presence of catastrophic risks.

We formulate our decision under uncertainty problem in Savage (1954) style, independent of the specific model considered in this paper, so that the results in this section are generally applicable. We fix a set $\mathcal{S}$ of states of nature and we let $\mathcal{A}$ denote a $\sigma$-algebra of subsets of $\mathcal{S}$. One state is the true state. We also fix a set $\mathcal{C}$ of consequences (outcomes, consumption) endowed with a $\sigma$-algebra $\mathcal{F}$. Since we are only interested in monetary outcomes, we may take $\mathcal{C}=\mathbb{R}_{+}$. A decision alternative (policy bundle) $X$ is a measurable mapping from $\mathcal{S}$ to $\mathcal{C}$, so that $X^{-1}(A) \in \mathcal{A}$ for all events $A \in \mathcal{F}$. We assume that the class of all decision alternatives $\mathcal{X}$ is endowed with a preference order $\succeq$.

DEFINITION 4.1 We say that expected utility (EU) holds if there exists a measurable and strictly increasing function $U: \mathcal{C} \rightarrow \mathbb{R}$ on the space of consequences, referred to as the utility function, and a probability measure $\mathbb{P}$ on $\mathcal{A}$, such that the preference order $\succeq$ on $\mathcal{X}$ is represented by a functional $V$ of the form $X \mapsto \int_{\mathcal{S}} U(X(s)) d \mathbb{P}=V(X)$. Thus, the decision alternative $X \in \mathcal{X}$ is preferred to the decision alternative $Y \in \mathcal{X}$ if, and only if, $V(X) \geq V(Y)$. 
In the Von Neumann and Morgenstern (1944) framework, utility $U$ is subjective, whereas the probability measure $\mathbb{P}$ associated with $\mathcal{A}$ is objective and known beforehand (decision under risk). In the more general framework of Savage (1954) adopted here, the probability measure itself can be, but need not be, subjective (decision under uncertainty).

Definition 4.2 We say that a risk $\epsilon: \mathcal{S} \rightarrow \mathbb{R}$ is heavy-tailed to the left (right) under $\mathbb{P}$ if its moment-generating function is infinite: $\mathrm{E}\left(e^{\gamma \epsilon}\right)=\infty$ for any $\gamma<0(\gamma>0)$.

Examples of heavy-tailed risks are the Student, lognormal, and Pareto distributions. Heavy-tailed risks provide appropriate mathematical models for low-probability high-impact events, such as environmental catastrophes.

Proposition 4.1 If EU is to discriminate univocally among all possible alternative outcome distributions, the utility function must be bounded.

Proposition 4.1 states that the EU functional is finite for all outcome distributions if, and only if, the utility function is bounded. Moreover, the axiomatization of EU is valid for all outcome distributions if, and only if, the utility function is bounded. The implications are non-trivial: boundedness of the utility function must hold not just in exotic situations but also in more familiar and economically relevant settings involving high levels of uncertainty. (See Moscadelli, 2004, regarding operational risk.)

In what follows we do not require the utility function to be bounded. We simply assume that the class of feasible outcome distributions is restricted (though the restriction may be void) in such a way that the utility function permits discrimination among them. Only a combination of utility function and outcome distribution that leads to finite expected utility is covered by the axiomatic justification of EU. Recall that $\operatorname{RRA}(x)=-x U^{\prime \prime}(x) / U^{\prime}(x)$ and $\operatorname{ARA}(x)=-U^{\prime \prime}(x) / U^{\prime}(x)$, and let

$$
\alpha^{*}=\inf _{x>0} \operatorname{RRA}(x), \quad \beta^{*}=\sup _{x>0} \operatorname{ARA}(x) .
$$

Now consider a representative agent with time-additive EU preferences and time-preference parameter $\rho>0$. We normalize (without loss of generality) the agent's consumption by setting $C_{0}=1$, and we define the pricing kernel (intertemporal marginal rate of substitution) as

$$
P\left(C_{1}^{*}\right)=\frac{U^{\prime}\left(C_{1}^{*}\right)}{(1+\rho) U^{\prime}(1)},
$$


where $C_{1}^{*}$ is optimal consumption at $t=1$. Consumption $C_{1}$ is commonly restricted to a budget-feasible consumption set which is subject to uncertainty $\left(\epsilon_{1}\right)$. We assume that the budget restriction takes the general form

$$
C_{1}^{*}\left(\epsilon_{1}\right) \leq B \exp \left(A \epsilon_{1}\right), \quad B, A>0,
$$

which need not be best-possible. (In our economy-climate model of Section 2, $B=e^{-\tau^{2} / 2} Y_{1} /\left(1+\xi H_{1}^{2}\right)$ and $A=\tau$. $)$ The expectation $\mathrm{E}(P)$ represents the amount of consumption in period 0 that the representative agent is willing to give up in order to obtain one additional certain unit of consumption in period 1.

The following result states that the expectation of the pricing kernel is finite for all outcome distributions whenever the concavity index (ArrowPratt index, index of absolute risk aversion) $\operatorname{ARA}(x)$ is bounded.

Proposition 4.2 Assume that EU holds and that the budget feasibility restriction (5) applies.

(a) If $\alpha^{*}>0$ and $\epsilon_{1}$ is heavy-tailed to the left under $\mathbb{P}$, then $\mathrm{E}(P)=\infty$;

(b) If $\beta^{*}<\infty$ and $\alpha^{*}=0$, then $\mathrm{E}(P)<\infty$ for any $\epsilon_{1}$.

If the EU maximizer has decreasing absolute risk aversion and increasing relative risk aversion, as is commonly assumed, a complete and elegant characterization of boundedness of the expected pricing kernel can be obtained, as follows.

Proposition 4.3 Assume that EU holds and that the budget feasibility restriction (5) applies. Assume furthermore that $R R A(x)$ exists and is nonnegative and non-decreasing for all $x \geq 0$ and that $A R A(x)$ is non-increasing for all $x>0$. Then, $\mathrm{E}(P)<\infty$ for any $\epsilon_{1}$ if and only if $\int_{0}^{\gamma} A R A(x) d x<\infty$ for some $\gamma>0$.

Notice that, when $\int_{0}^{\gamma} \operatorname{ARA}(x) d x=\infty$ for some $\gamma>0$, both $\alpha^{*}>0$ and $\alpha^{*}=0$ can hold. If $\alpha^{*}>0$ then we do not need the full force of Proposition 4.3; it is sufficient that $\epsilon_{1}$ is heavy-tailed to the left. Then $\mathrm{E}(P)=\infty$ by Proposition 4.2(a). If $\alpha^{*}=0$ then heavy-tailedness alone is not sufficient, but we can always find an $\epsilon_{1}$ such that $\mathrm{E}(P)=\infty$. When $\int_{0}^{\gamma} \operatorname{ARA}(x) d x=\infty$ then $\beta^{*}=\infty$. But when $\int_{0}^{\gamma} \operatorname{ARA}(x) d x<\infty$, both $\beta^{*}<\infty$ and $\beta^{*}=\infty$ can occur.

The above propositions provide necessary and sufficient conditions on the utility function to ensure that expected utility and expected marginal utility (hence the expected pricing kernel) are finite, also in the presence of heavy tails. These compatibility results are generally applicable to standard multiperiod welfare maximization problems. The importance of the results lies in 
the fact that (i) if expected utility is infinite, the axiomatic justification of $\mathrm{EU}$ is not valid, and (ii) if the expected pricing kernel is infinite, then the amount of consumption in period 0 which the representative agent is willing to give up in order to obtain one additional certain unit of consumption in period 1 is infinite, which is not credible.

\section{$5 \quad$ A two-period model}

In Sections 2-4 we assumed an infinite horizon. We now specialize to two periods. The two-period model captures the essence of our problem while remaining numerically tractable in the presence of uncertainty.

If the policy maker has a (finite) $T$-period policy horizon, then we write welfare as

$$
W=\sum_{t=0}^{T-1} \frac{L_{t} U\left(x_{t}\right)}{(1+\rho)^{t}}+\frac{1}{(1+\rho)^{T}} \sum_{t=0}^{\infty} \frac{L_{T+t} U\left(x_{T+t}\right)}{(1+\rho)^{t}},
$$

where $x_{t}=C_{t} / L_{t}$ denotes per capita consumption in period $t$. If $\left\{x_{t}^{*}\right\}$ denotes the optimal path for $\left\{x_{t}\right\}$, then we define the scrap value as

$$
S_{T}=\sum_{t=0}^{\infty} \frac{L_{T+t} U\left(x_{T+t}^{*}\right)}{(1+\rho)^{t}} .
$$

Maximizing $W$ is then equivalent to maximizing

$$
\sum_{t=0}^{T-1} \frac{L_{t} U\left(x_{t}\right)}{(1+\rho)^{t}}+\frac{S_{T}}{(1+\rho)^{T}}
$$

The scrap value $S_{T}$ will depend on the state variables at time $T$, in particular $K_{T}$ and $M_{T}$, and this functional relationship is the scrap value function: $S_{T}=S\left(K_{T}, M_{T}\right)$. If $T$ is large we may ignore the scrap value $S_{T}$ because of the large discount factor $(1+\rho)^{T}$. But if $T$ is small, then we need to model $S_{T}$ explicitly, thus emphasizing the fact that the policy maker has the double objective of maximizing discounted welfare over a finite number of periods $T$, while also leaving a reasonable economy for the next policy maker, based on the remaining capital stock and $\mathrm{CO} 2$ concentration.

The simplest approximation to $S_{T}$ is the linear function

$$
S_{T}=\nu_{0}+\nu_{1} K_{T}-\nu_{2} M_{T} \quad\left(\nu_{1}>0, \nu_{2}>0\right)
$$

where $\nu_{1}$ and $\nu_{2}$ denote the scrap prices of capital and pollution at the beginning of period $T$. This scrap value function captures the idea that the 
next government will be happier if there is more capital and less pollution at the beginning of its policy period. But the linear scrap value function has some problems. We shall therefore introduce nonlinear scrap value functions, whose specific form depends on the form of the utility function; see our background document Ikefuji et al. (2011b) for further details on our treatment of scrap value functions.

The simplest version of the model occurs when $T=2$ in which case we have only two periods. We can write welfare in this case as

$$
W=W\left(\mu_{0}, C_{0}, \mu_{1}, C_{1}, \epsilon_{1}\right)=W_{0}+\frac{W_{1}}{1+\rho}+\frac{S_{2}}{(1+\rho)^{2}} .
$$

The policy restrictions (1) are explicitly imposed, so that we maximize a restriction of expected welfare; see Appendix A. Randomness results from $d_{1}$ only, because the temperature-impact $d_{0}$ at the beginning of period 0 is known to us (we set $\bar{d}_{0}=1$, equal to its expectation), and $d_{2}$ at the end of period 1 does not appear in the welfare function. Hence, the only source of randomness is caused by the error $\epsilon_{1}$. The policy maker has to choose the policy bundles $\left(C_{0}, I_{0}, \mu_{0}\right)$ at the beginning of period 0 and $\left(C_{1}, I_{1}, \mu_{1}\right)$ at the beginning of period 1 that will maximize expected welfare.

Realizing that at the beginning of period 1 the temperature-impact $d_{1}$ is observed based on the realization of $\epsilon_{1}$, the policy maker will maximize expected welfare in three steps as follows. First, he/she maximizes welfare $W=W\left(\mu_{0}, C_{0}, \mu_{1}, C_{1}, \epsilon_{1}\right)$ with respect to $\left(\mu_{1}, C_{1}\right)$ conditional on $\left(\mu_{0}, C_{0}, \epsilon_{1}\right)$ and under the restriction (1). This gives $\left(\mu_{1}^{*}, C_{1}^{*}\right)$ and concentrated welfare

$$
W^{*}\left(\mu_{0}, C_{0}, \epsilon_{1}\right)=W\left(\mu_{0}, C_{0}, \mu_{1}^{*}, C_{1}^{*}, \epsilon_{1}\right) .
$$

Then the expectation $\bar{W}\left(\mu_{0}, C_{0}\right)=\mathrm{E}\left(W^{*}\left(\mu_{0}, C_{0}, \epsilon_{1}\right)\right)$ is computed, if it exists. Finally, $\bar{W}$ is maximized with respect to $\left(\mu_{0}, C_{0}\right)$.

\section{Compatibility}

We now have a simplified Nordhaus model with Weitzman-type uncertainty in a two-period framework. In Table 2 we present the optimal values of the policy and other selected variables obtained from maximizing expected welfare. (Our background document contains the complete tables.) The results allow for uncertainty, consider the short run (two periods) rather than the long run (sixty periods), and also take scrap values into account.

We need values for the exogenous variables $L_{t}, A_{t}, \sigma_{t}$, and $\psi_{t}$; these are given in our background document. We note that $Y_{0}=556.67$ and 


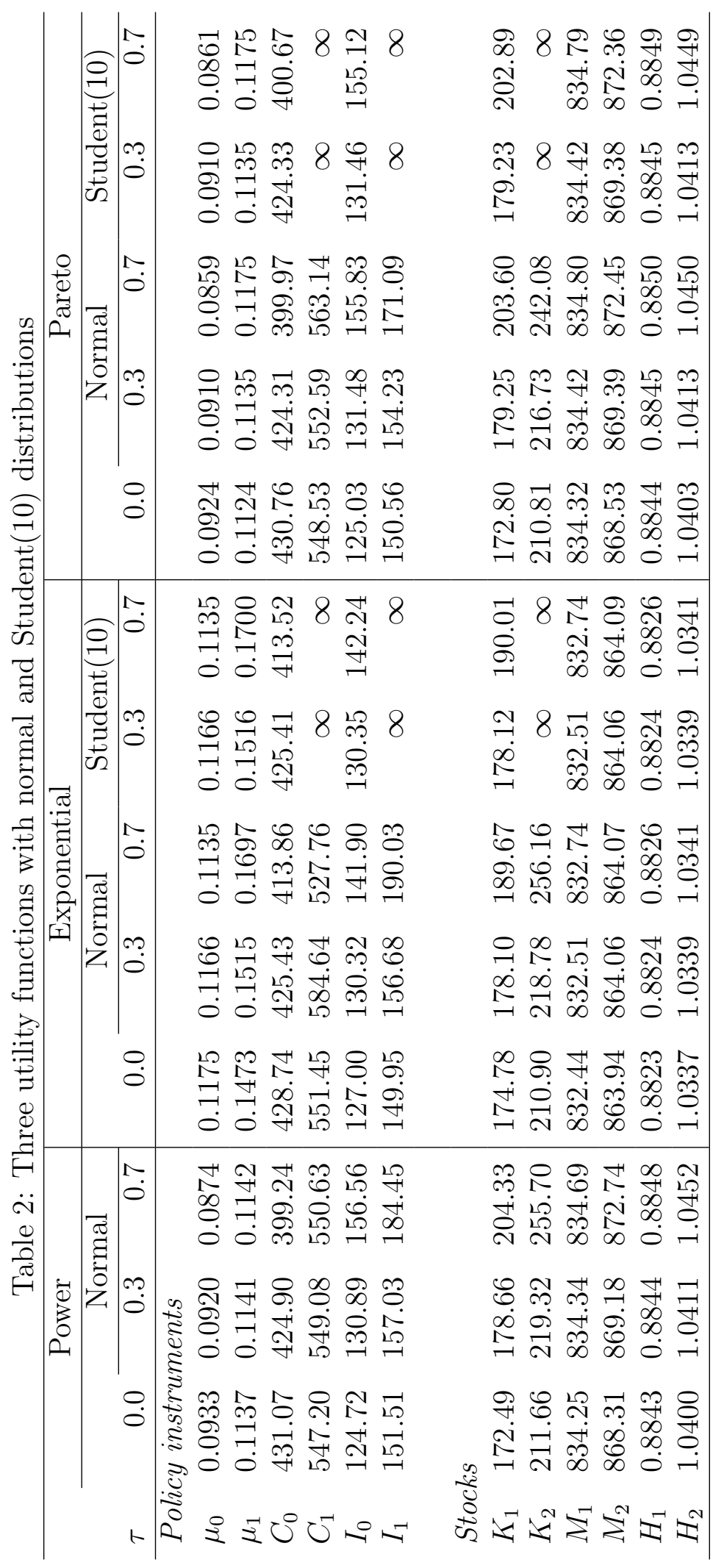


$d_{0}=0.9985$ are constant over different scenarios and functions, and that the values of $\mu_{0}, C_{0}, I_{0}, E_{0}, \omega_{0}, K_{1}, M_{1}$, and $H_{1}$ are optimal values. In contrast, $\mu_{1}, C_{1}, I_{1}, Y_{1}, E_{1}, \omega_{1}, d_{1}, K_{2}, M_{2}$, and $H_{2}$ are optimal functions of $\epsilon_{1}$. What we present in the tables are their expectations.

We consider three utility functions (power, exponential, Pareto) and two distributions (normal, Student). First, power utility as given in (2) for $\alpha=2$ : $U(x)=1-1 / x$. The following proposition states that if the random errors $\epsilon_{t}$ are generated by a normal $\mathrm{N}(0,1)$ distribution, then the expectation of welfare exists for power utility, but if we move one step away from normality and assume a Student distribution with any finite degrees of freedom, then the expectation does not exist. It illustrates the consequences of violating the conditions of Proposition 4.1.

Proposition 6.1 With power utility, expected welfare exists under normality but not under a Student distribution.

It follows that the much-used power utility function is incompatible with expected utility theory with heavy tails, not because utility theory itself is at fault but because power utility is inappropriate when tails are heavy.

Motivated by the conditions derived in Section 4 and by the fundamental insight that the economic model and the statistical model must be compatible, and because we wish to leave distributional assumptions unrestricted at this stage, we consider two alternative utility functions: the exponential function and the 'Pareto' function. Other choices are permitted but may require restrictions on distributional assumptions. The exponential utility function is given by

$$
U(x)=1-e^{-\beta x} \quad(\beta>0)
$$

with $\operatorname{ARA}(x)=\beta$ and $\operatorname{RRA}(x)=\beta x$, and the Pareto utility function by

$$
U(x)=1-\left(\frac{\lambda}{x+\lambda}\right)^{k} \quad(k>0, \lambda>0)
$$

with $\operatorname{ARA}(x)=(k+1) /(x+\lambda)$ and $\operatorname{RRA}(x)=(k+1) x /(x+\lambda)$. The Pareto function was proposed in Ikefuji et al. (2011a), where it is also shown that this function enjoys a combination of appealing properties especially relevant in heavy-tailed risk analysis. We choose the parameters as follows: exponential function $(\beta=25)$, and Pareto function $(k=1.5, \lambda=0.02)$. This choice of parameters is determined by the point $x^{*}$, where we want the three utility functions to be close. Suppose we want the functions to be close at $x^{*}=0.08$, which is approximately the value of $C_{0} / L_{0}$ and $C_{1} / L_{1}$. Then, given that $\alpha=2$, we find $\beta=2 / x^{*}=25$, and, for any $k>1, \lambda=(k-1) x^{*} / 2$. 
The power function is unbounded from below, hence violates the conditions of Proposition 4.1, and has constant and positive RRA, hence violates the conditions of Proposition 4.3. Both the exponential and the Pareto function are bounded from above and below, hence satisfy the conditions of Proposition 4.1. The exponential function has constant and positive ARA, hence satisfies the conditions of Proposition 4.3, while the RRA is unbounded for large $x$. In contrast, the RRA in the Pareto function is bounded between 0 and $k+1$, and it further satisfies $\operatorname{RRA}(0)=0$ and $\operatorname{ARA}(0)<\infty$, hence satisfies the conditions of Proposition 4.3. Notice that the fact that $\operatorname{RRA}(0)=0$ (as is the case for the exponential and the Pareto utility functions) does not imply that the representative agent is risk-neutral at $x=0$. In particular, we have $\operatorname{ARA}(0)=\beta$ for the exponential function and $\operatorname{ARA}(0)=(k+1) / \lambda$ for the Pareto function.

\subsection{Results for power utility}

The first panel of Table 2 gives the results for power utility. For $\tau=0$ there is no uncertainty. For $\tau>0$ there is uncertainty, and all policy variables are affected when $\tau$ increases. More uncertainty results in less abatement, less consumption, and more investment in period 0 , and to more abatement, consumption, and investment in period 1 . In period 1 , the changes in abatement and consumption are negligible. The increase in $I_{0}$ with $\tau$ can be explained by precautionary savings. The restriction on $I_{1}$ can be viewed as a penalty for negative investment. To avoid this penalty, the policy maker can increase the budget in period 1 by investing more in period 0 . As the amount of uncertainty increases, the probability of negative investment increases, ceteris paribus. In response, the policy maker increases investment at the expense of abatement and consumption in period 0 . The increase in $I_{0}$ leads to higher output in period 1 , which explains the increases in $I_{1}, K_{2}$, $M_{2}$, and $H_{2}$. The decrease in $\mu_{0}$ leads to higher emissions in period 0 , and increases carbon concentration and temperature in period 1. An additional reason why investment in period 1 increases with uncertainty is that positive shocks translate into possibly unlimited upward shocks in $I_{1}$, but negative shocks will never cause $I_{1}$ to drop below zero.

\subsection{Results for exponential and Pareto utility}

Turning now to the alternative utility functions, we first maximize (deterministic, hence $\tau=0$ ) welfare over sixty periods (600 years) for both exponential and Pareto utility. A selection of the resulting optimal values is shown in Table 3. When we compare the results with those in Table 1, we see that the 
Table 3: Comparison of stocks in Exponential and Pareto models

\begin{tabular}{rrrrrrrrr}
\hline & \multicolumn{2}{c}{2005} & \multicolumn{2}{c}{2055} & \multicolumn{2}{c}{2105} & \multicolumn{2}{c}{2155} \\
& Expo & Pareto & Expo & Pareto & Expo & Pareto & Expo & Pareto \\
\hline$K$ & 137 & 137 & 286 & 343 & 388 & 666 & 456 & 1220 \\
$M$ & 809 & 809 & 1012 & 993 & 1328 & 1258 & 1727 & 1512 \\
$H$ & 0.7 & 0.7 & 1.6 & 1.5 & 2.6 & 2.5 & 3.7 & 3.3 \\
\hline
\end{tabular}

optimal stock values from the Pareto function closely resemble the optimal stock values from the power function, but not those from the exponential function. In contrast to power and Pareto, where RRA flattens out, the RRA for the exponential distribution continues to increase, and hence the growth rate of marginal utility continues to increase as well. As $x$ increases, consumption will therefore increase, and investment and abatement will decrease. As a result, $C / Y$ is relatively large for exponential utility. The low growth rate of capital (for exponential utility) leads to a low growth rate of output. However, since more consumption leads to less abatement, the growth rate of $\mathrm{CO} 2$ concentration is high even when the amount of production is low. Consequently, $M$ and $H$ are high compared to power and Pareto. When $x<x^{*}$, RRA (Pareto) is close to RRA (exponential), so that more is consumed and less invested when the Pareto function is used instead of the power function. But when $x>x^{*}$, RRA (Pareto) is close to RRA (power). The optimal path of $K$ is slightly lower and the optimal paths of $M$ and $H$ are slightly higher for Pareto than for power utility.

Since exponential utility is calibrated to be close to power utility at $x=x^{*}$, the two-period results for the two utility functions do not differ greatly; see the second panel of Table 2. This is especially true for $\tau=0$, where only the abatement fraction $\mu$ is higher for exponential utility, and therefore temperature $H$ is lower. When $\tau$ increases, $I_{0}$ increases less and $I_{1}$ increases more for exponential than for power. Moreover, as the uncertainty parameter $\tau$ increases, $M_{2}$ does not change much in the exponential case, while it increases in the power case. The effect of uncertainty on the marginal scrap values is therefore larger in the exponential case than in the power case. As in the first panel (power utility), more uncertainty results in less abatement, less consumption, and more investment in period 0 , and to more abatement, and investment in period 1 .

Suppose next that the underlying distribution has heavier tails: Student instead of normal. Under power utility, expected welfare does not exist any more. But under bounded utility, expected welfare always exists. Although the effect of the excess kurtosis on expected welfare is large and discontinuous, the effect on the optimal values is relatively small. This is good, because the 
Student distribution with 10 degrees of freedom is in fact quite close to the normal distribution as Figure 1 reveals, and hence it would be unreasonable if a 'small' change in distributional assumptions would lead to a large possibly 'discontinuous' change in optimal policies.

All variables move in the same direction as before when $\tau$ increases. Notice that some variables $\left(C_{1}, I_{1}\right.$, and $\left.K_{2}\right)$ have infinite expectations even though expected welfare is finite. This is no surprise because these variables are unbounded and depend on $\bar{d}_{1}=e^{-\tau^{2} / 2} e^{\tau \epsilon_{1}}$. When $\epsilon_{1}$ follows a Student distribution, $\mathrm{E}\left(\bar{d}_{1}\right)=\infty$ and this property carries over to the other three variables.

Finally we consider the third utility function, Pareto utility. The optimal values are presented in the third panel of Table 2. We would expect that Pareto and power are relatively close in the observed data range. This is indeed the case as a comparison of the first and third panels reveals. There is little difference between the two panels in the case of no uncertainty, and also when $\tau$ increases. The effect of excess kurtosis is again small, as it should be.

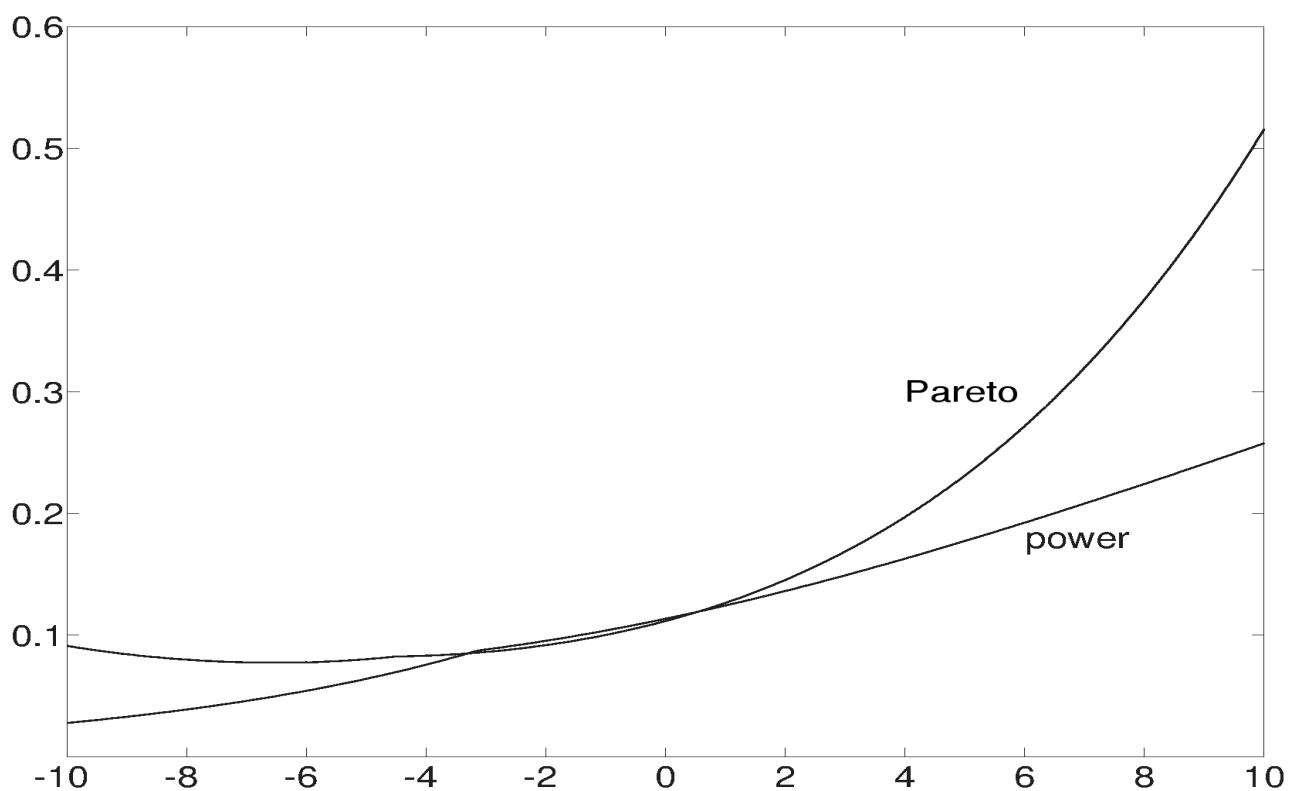

Figure 2: $\mu_{1}^{*}$ as a function of $\epsilon_{1}$ : Pareto versus power utility

The important difference between power and Pareto utility is only revealed when low levels of per capita consumption become relevant, that is, in near-catastrophe cases. This is clarified in Figure 2 , where we present $\mu_{1}^{*}$ as a function of $\epsilon_{1}$ for $\tau=0.3$. The expected value of $\mu_{1}^{*}$ is 0.1141 for power 
utility under normality (first panel), and 0.1135 for Pareto utility under either normality or Student(10) (third panel). This is not very different. But for values of $\epsilon_{1}$ further away from 0 the difference is large.

\section{Learning, agreement and robustness}

\subsection{Learning and agreement}

To complete the model we need to specify our input parameters. We now show, in a stylized setting, how this can be achieved in a process towards agreement, using experts' priors. The key to this learning and agreement process is the translation of model parameters that are relatively difficult to interpretinto quantities that allow a more straightforward interpretation, hence are easier to specify.

Our parameters cannot be estimated using conventional methods and historical data, but experts will have prior ideas about these parameters. Different experts will have different priors. Model output can be generated on the basis of various priors. Then, in an iterative procedure, one learns about the parameter values from experts' opinions and model output, and an agreeable intersection of model parameters may be reached.

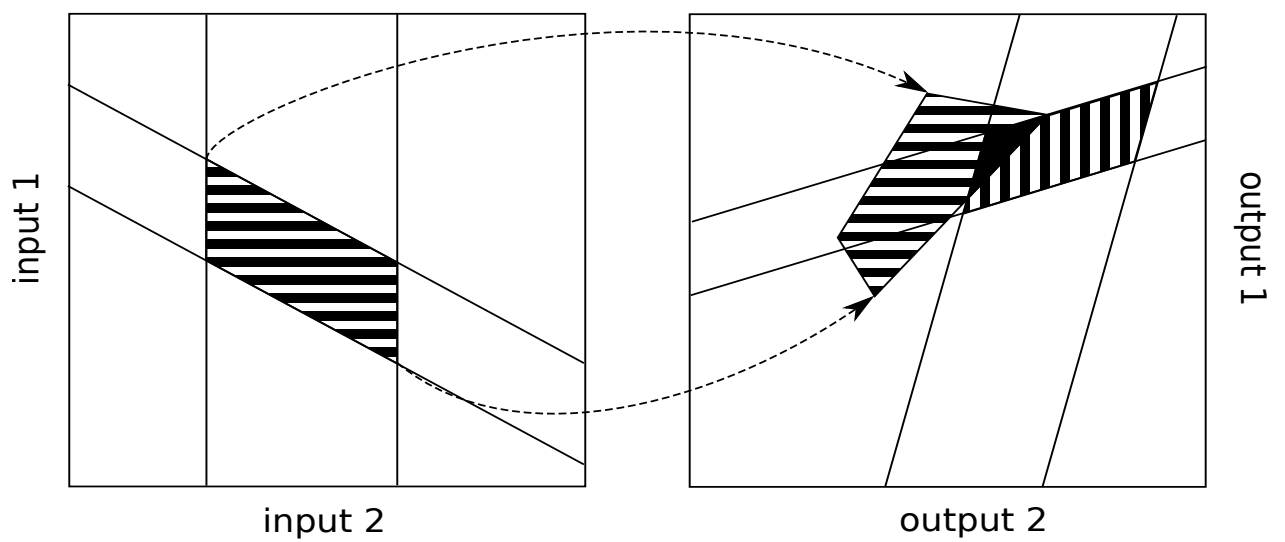

Figure 3: The decision making process

This process is illustrated in Figure 3. In the left panel, we visualize the contributions of two experts. One expert states that the value of input 2 should be bounded as indicated by the two vertical lines. The other expert provides a lower and upper bound for the value of input 1, depending on the value of input 2. The horizontally-shaded area gives the combinations 
of inputs that are acceptable to both experts. The right panel is more complicated. We first visualize the contributions of two policy makers regarding two output variables. This is the vertically-shaded area, giving the combinations of outputs that are acceptable to both policy makers. Next we map the left panel onto the right panel. For every acceptable combination of inputs the model provides one combination of outputs, that is, one point in the right panel. The horizontally-shaded area in the right panel is the image of the horizontally-shaded area in the left panel. We now have two areas in the right panel: the vertically-shaded area and the horizontally-shaded area. If the two areas do not intersect, then the experts and policy makers must adjust their priors in an iterative process of learning. Once the areas do intersect, agreement is possible. The black triangle then contains all points for which both inputs and outputs are acceptable. Agreement must be reached on the three policy variables $\left(\mu_{0}, C_{0}, I_{0}\right)$, and we recall that expected welfare is maximized in three steps as described at the end of Section 5, yielding the optimal policy $\left(\mu_{0}^{*}, C_{0}^{*}, I_{0}^{*}\right)$.

\subsubsection{Inputs}

Our analysis requires prior beliefs about various inputs, in particular: form of the utility function (Pareto or otherwise), degree of risk-aversion $(k, \lambda)$, discount rate $(\rho)$, form of the distribution (Student or otherwise), and volatility $(\tau)$. If agreement is to be reached, then the policy makers must be willing to adjust their individual priors on each of these inputs, based on the experts' opinions and the generated output.

We want a distribution which allows heavy tails (Ursúa, 2010), such as the Student distribution (Weitzman, 2009). Given our treatment of stochasticity, power utility is not compatible with the Student distribution, because the required expectations don't exist. Also, exponential utility has the disadvantage that RRA increases without bound. Pareto utility provides a useful compromise: it exhibits exponential-like features when per capita consumption is small, and power-like features otherwise (Ikefuji et al., 2011a). We therefore confine ourselves to Pareto utility, assume that $\epsilon_{1}$ follows a Student distribution, and take the following parameter values as our benchmark:

$$
k=1.5, \quad \lambda=0.02 \quad \tau=0.3, \quad \text { df }=10, \quad \rho=0.1605 .
$$

Note that the value of $\lambda$ is linked to $k$ through $\lambda=0.04(k-1)$, as explained in Section 6. Priors regarding risk aversion are based on our background document. The symbol df denotes the degrees of freedom in the Student distribution, and the discount rate of 0.1605 per decade corresponds to an annual discount rate of 0.015 . 
Table 4: Parameter calibration based on Pareto utility and Student distribution

\begin{tabular}{|c|c|c|c|c|c|c|c|}
\hline & \multicolumn{4}{|c|}{ Agreement } & \multicolumn{3}{|c|}{ Robustness } \\
\hline & $a$ & $b$ & $c$ & $d$ & $e$ & $f$ & $g$ \\
\hline \multicolumn{8}{|c|}{ Parameter values } \\
\hline$\tau$ & 0.3 & 0.3 & 0.5 & 0.3 & 0.5 & 0.7 & 0.5 \\
\hline df & 10 & 25 & 10 & 10 & 25 & 10 & 10 \\
\hline$k$ & 1.5 & 1.5 & 1.5 & 2.0 & 1.5 & 1.5 & 2.0 \\
\hline \multicolumn{8}{|c|}{ Policy instruments, beginning of period 0} \\
\hline$\mu_{0}$ & 0.0910 & 0.0910 & 0.0888 & 0.1192 & 0.0887 & 0.0861 & 0.1163 \\
\hline$C_{0}$ & 424.33 & 424.31 & 413.71 & 438.01 & 413.50 & 400.67 & 427.56 \\
\hline$I_{0}$ & 131.46 & 131.47 & 142.08 & 117.73 & 142.29 & 155.12 & 128.19 \\
\hline \multicolumn{8}{|c|}{ Capital stock and expectations } \\
\hline$K_{1}$ & 179.23 & 179.25 & 189.86 & 165.50 & 190.06 & 202.89 & 175.96 \\
\hline$\mu_{1}$ & 0.1135 & 0.1135 & 0.1154 & 0.1604 & 0.1154 & 0.1175 & 0.1655 \\
\hline$H_{2}$ & 1.0413 & 1.0413 & 1.0429 & 1.0309 & 1.0430 & 1.0449 & 1.0323 \\
\hline \multicolumn{8}{|c|}{ Probabilities of catastrophe $\pi_{\ell}$} \\
\hline$\pi_{a}$ & $5.0 \mathrm{E}-03$ & $3.3 \mathrm{E}-03$ & $5.2 \mathrm{E}-02$ & $5.1 \mathrm{E}-03$ & $5.3 \mathrm{E}-02$ & $1.4 \mathrm{E}-01$ & $5.2 \mathrm{E}-02$ \\
\hline$\pi_{b}$ & $2.3 \mathrm{E}-05$ & $5.9 \mathrm{E}-07$ & $1.4 \mathrm{E}-03$ & $2.6 \mathrm{E}-05$ & $5.5 \mathrm{E}-04$ & $1.2 \mathrm{E}-02$ & $1.5 \mathrm{E}-03$ \\
\hline$\pi_{c}$ & $2.5 \mathrm{E}-07$ & $5.0 \mathrm{E}-11$ & $2.8 \mathrm{E}-05$ & $2.6 \mathrm{E}-07$ & $8.2 \mathrm{E}-07$ & $4.9 \mathrm{E}-04$ & $3.0 \mathrm{E}-05$ \\
\hline \multicolumn{8}{|c|}{ Values of statistical subsistence $V_{\ell}=V S S_{\ell} / C_{0}$} \\
\hline$V_{a}$ & $2.8 \mathrm{E}+01$ & $2.9 \mathrm{E}+01$ & $4.8 \mathrm{E}+00$ & $2.4 \mathrm{E}+01$ & $4.1 \mathrm{E}+00$ & $2.9 \mathrm{E}+00$ & $4.1 \mathrm{E}+00$ \\
\hline$V_{b}$ & $1.3 \mathrm{E}+04$ & $2.8 \mathrm{E}+05$ & $3.1 \mathrm{E}+02$ & $1.1 \mathrm{E}+04$ & $5.3 \mathrm{E}+02$ & $5.2 \mathrm{E}+01$ & $2.6 \mathrm{E}+02$ \\
\hline$V_{c}$ & $1.9 \mathrm{E}+06$ & $4.2 \mathrm{E}+09$ & $2.0 \mathrm{E}+04$ & $1.6 \mathrm{E}+06$ & $3.6 \mathrm{E}+05$ & $1.4 \mathrm{E}+03$ & $1.7 \mathrm{E}+04$ \\
\hline
\end{tabular}

Our benchmark is column $a$ in Table 4 . The model outputs are within credible bounds: policy variables at the beginning of period $0\left(\mu_{0}, C_{0}, I_{0}\right)$; stock variables at the beginning of period $1\left(K_{1}\right.$ and also $M_{1}=834.42$ and $\left.H_{1}=0.8845\right)$; and expectations $\left(\mathrm{E}\left(\mu_{1}\right), \mathrm{E}\left(H_{2}\right)\right.$, and also $\left.\mathrm{E}\left(M_{2}\right)=869.38\right)$. If we consider temperature $H_{2}$ as a function of $\epsilon_{1}$ we find relatively low volatility in comparison to the confidence intervals proposed by the IPCC (2007, Chapter 10). The reason for this is twofold. First, the IPCC determines confidence intervals by considering multiple deterministic climate models, not a single stochastic one as we do. Second and more importantly, the IPCC confidence intervals are based on non-mitigation scenarios, while our model takes policy effects into account. For both reasons, the volatility in temperature found by the IPCC is higher than what we find. 


\subsubsection{Outputs}

In addition to the 'direct' outputs of our model we also have 'derived' outputs, in particular the probability of catastrophe. These derived outputs are functions of the direct outputs and they represent important policy variables on which prior information is available. Hence, they also require agreement.

We propose to define catastrophe as the event $C_{1}^{*} \leq \underline{C}$ for some given value $\underline{C}>0$. The probability of catastrophe is then given by $\pi=\operatorname{Pr}\left(C_{1}^{*} \leq\right.$ $\underline{C})$. We shall consider three different values of $\underline{C}: \underline{C}_{a}, \underline{C}_{b}$, and $\underline{C}_{c}$, corresponding to three levels of catastrophe, labeled $A, B$, and $C$. Catastrophe $A$ occurs when $20 \%$ of the world population live in extreme poverty, and catastrophes $B$ and $C$ occur when $50 \%$ and $80 \%$ of the world population live in extreme poverty, respectively. The definitions and priors proposed in this subsection are based on background material provided in Ikefuji et al. (2011b).

We must agree on acceptable values for the probability $\pi$ of catastrophe. We have studied acceptable risks in various situations, and we conclude that an acceptable probability for an economy-climate catastrophe in the next 10 -year period is in the range $10^{-5}-10^{-6}$. Given the definition of catastrophe we propose: $\pi_{a}=0.1, \pi_{b}=0.001$, and $\pi_{c}=0.00001$ as reasonable values.

In the benchmark model we find $\pi_{a}=0.005, \pi_{b}=0.00002$, and $\pi_{c}=$ 0.0000003 , which is much lower than the acceptable values. Given the associated costs, it seems unnatural that policies would be chosen that mitigate the probability of a global economy-climate catastrophe far beyond acceptable levels. What can we do about this? One possibility is to make the tails heavier or lighter, that is, to adjust the degrees of freedom. If we set $\mathrm{df}=25$ then $\pi$ becomes even smaller. In general, $\pi$ becomes smaller as the tails become lighter ( $\mathrm{df}$ increases), as one would expect. For $\mathrm{df}=\infty$ (the normal distribution) we find $\pi_{a}=2.3 \mathrm{E}-03, \pi_{b}=5.3 \mathrm{E}-10$, and $\pi_{c}=1.5 \mathrm{E}-24$. Interestingly, the policy variables are hardly affected (column $b$ ), not even when $\mathrm{df}=200$ or $\mathrm{df}=\infty$. If we set $\mathrm{df}=3$, which is the minimum value where $\operatorname{var}\left(\epsilon_{1}\right)$ exists, then $\pi_{a}=0.008$, a little higher than for $\mathrm{df}=10$, but not enough. So, adjusting the degrees of freedom hardly changes the results.

Perhaps the fact that the heaviness of the tail (degrees of freedom) has little effect on the optimal policy is caused by the Pareto utility function. Maybe this function does not distinguish well between different tails? In fact, this is not so. It follows from Figure 1 (and Section 6) that $\tau$ has much more impact than df. Hence the Pareto function does distinguish between different tails.

Perhaps we should then adjust the value of $\tau$. In our benchmark we set $\tau=0.3$ as a reasonable starting point. We could revise $\tau$ upwards. We 
argued in Section 3 and Figure 1 that $\tau=0.7$ is an upper bound to the volatility. Let us therefore consider the case $\tau=0.5$. A larger value of $\tau$ means more volatility and hence one would expect less consumption and more investment. This is indeed what happens (column $c$ ). Also, the probabilities are affected and are now much closer to our prior ideas.

We can also adjust the curvature $k$ (and $\lambda$ ). If $k$ increases, then agents become less risk-averse and, as expected, there is more consumption and less investment (column $d$ ). The probabilities are not much different from our benchmark in $a$, but the values of $\mu_{0}$ and $\mu_{1}$ are very high and the capital stock accumulation rate is only $1.9 \%$ per year, which is too low.

Finally, we could adjust the discount rate $\rho$. This is an important issue (see, for example, Gollier, 2002, 2008, and the references therein), with possibly significant (yet not 'discontinuous') impact on the optimal policies. It is, however, beyond the scope of this study.

Based on these comparisons it seems that policy $c$ should be recommended. There is, however, one other derived output which is often discussed, namely the value of statistical life. If we agree on the definition of catastrophe, then we can also define the 'value of a statistical subsistence' (VSS) as the amount of consumption in period 0 that the government is willing to trade off in order to change the probability of catastrophe; see Ikefuji et al. (2011b) for further details. The VSS is similar to the value of statistical life (VSL), except that it refers to the condition of just having enough food to stay alive (more than $\$ 1$ day) rather than to life. We propose $\mathrm{VSS}_{a}=C_{0}, \mathrm{VSS}_{b}=10 C_{0}$, and $\mathrm{VSS}_{c}=100 C_{0}$ as reasonable orders of magnitude. The VSS (and the VSL) is a difficult concept to measure, and the VSS priors (Ikefuji et al., 2011b) may be unreliable. As such it should not carry too much weight as a derived output. Still we notice that the VSSs of our preferred policy $c$ are much closer to our reasonable values than the VSSs in columns $a, b$, and $d$.

\subsection{Robustness}

If we believe that column $c$ is the best, then we should do some further robustness checks, starting from column $c$ rather than column $a$. We have done extensive robustness checks and some representative results of this analysis is reported in columns $e^{-}-g$ of Table 4; see Ikefuji et al. (2011b) for further results. If we adjust the degrees of freedom (column $e$ ), then not much happens. There is little to choose between columns $c$ and $e$. The optimal policy $\left(\mu_{0}^{*}, C_{0}^{*}, I_{0}^{*}\right)$ is hardly affected, which is a good thing, because it means that our policy is not too sensitive to changes in the heaviness of the tail (degrees of freedom). In column $f$ we consider $\tau=0.7$. Here the probabilities of 
catastrophe seem to be too large. For example, we have $\pi_{c}=0.0005$ and it is doubtful if the government would find this acceptable. The choice of volatility $\tau$ does, however, affect the policy, and hence is important. In column $g$ we adjust the curvature of the Pareto utility function. The probabilities are hardly affected but there will be more consumption, less investment, and in particular more (perhaps too much) abatement. On the basis of these and other robustness checks we conclude that policy $c$ is robust against small changes in the underlying assumptions and parameter values.

\section{Concluding remarks}

Our strategy in this paper has been to specify a stochastic economy-climate model, building on Nordhaus' deterministic economy-climate model while allowing for Weitzman-type stochasticity. We show that, under expected power utility, the model is fragile with respect to distributional assumptions. Based on general results regarding the relationship between the richness of the class of utility functions and the generality of the permitted distributional assumptions, we restrict ourselves to utility functions that are compatible with our distributional assumptions. Thus we avoid the unacceptable conclusion that society should sacrifice an unlimited amount of consumption to reduce the probability of catastrophic climate change by even a small amount. After reaching agreement on the model parameters, a sensitivity analysis shows that our completed model and the resulting optimal policies are quite robust and sensibly sensitive.

The fragility of the model under expected power utility to heavy-tailed distributional assumptions is not unexpected. Weitzman (2009) summarizes this fragility and the perceived non-existence of a robust solution in a 'dismal theorem'. While we agree with Weitzman that incompatible pairs of utility functions and distribution functions exist, our objections to the dismal theorem are twofold. First, the result is implied by using an incompatible (invalid) model specification. A key ingredient in Weitzman's model is the power utility function. This popular utility function is characterized by constant relative risk aversion (CRRA). The assumption of CRRA, hence $\operatorname{RRA}(0)>0$, is not appropriate when dealing with extremely low levels of consumption, and it is exactly the behavior at these low consumption levels that leads to the dismal theorem. As we have demonstrated formally in Section 4 and numerically in Section 6, Weitzman's result is avoided when the economic model (utility function) is compatible with the statistical model (heavy tails).

Second, more effort can be made to know an input parameter that is 'hard 
to know', and we have described a (stylized) learning-and-agreement procedure for precisely this purpose in Section 7. Although it is difficult to state upper and lower bounds for the 'VSL-like' input parameter of Weitzman, we can still obtain reasonable constraints on difficult-to-know parameters of interest indirectly. The economic model translates the parameter of interest into output variables with an easier interpretation (such as the optimal policies and the probability of catastrophe). Bounds on these output variables, together with the economic model, imply bounds on the input parameter of interest.

Much of the analysis in our paper is not limited to extreme climate change. A similar analysis could apply in other policy making settings involving catastrophic risks, such as the development of new financial incentive schemes to mitigate the risk of extreme systemic failures and resulting financial economic crises, or policies concerning medical risks (pandemic flu and vaccination risks).

Let us finally admit four limitations of our paper, and indicate possible generalizations. First, in Section 6 we have focussed our attention on bounded utility functions, so as to avoid having to restrict distributional assumptions. In general, one could assume more structure on stochasticity (yet still allow for heavy tails) and broaden the constraints on utility. In particular, unbounded utility (such as HARA with $0<\alpha \leq 1$ ) is also permitted under additional assumptions on stochasticity. Second, for simplicity and clarity of presentation, we have restricted our numerical analysis to only two periods. Conceptually, much of our analysis will remain intact when considering more than two periods. Third, to account for the fact that the policy maker has the double objective of maximizing current consumption, while also leaving a reasonable economy for the next policy maker, we have used scrap values in our analysis. We ignore, however, stochasticity in the scrap value function after the second period. The development of a numerically tractable economy-climate model with multi-period stochasticity and stochasticity in scrap values after the last period is a subject for further research. Finally, the equations making up our stochastic economy-climate model are of a simple and stylized nature, and each one of them, including the specification of stochasticity, leaves room for generalizations and extensions. 


\section{Appendices}

\section{A Kuhn-Tucker conditions under positive in- vestment}

Consider the economy-climate model of Section 2 in a two-period set-up. Let $U$ be a general well-behaved utility function and let $S^{(1)}$ and $S^{(2)}$ be general well-behaved scrap value functions. At the beginning of period 1 our welfare function, conditional on $\left(C_{0}, \mu_{0}, \epsilon_{1}\right)$, is

$$
W=L_{1} U\left(C_{1} / L_{1}\right)+\nu_{1} S^{(1)}\left(K_{2}\right)-\nu_{2} S^{(2)}\left(M_{2}\right) .
$$

We have four constraints: $C_{1} \geq 0, I_{1} \geq 0, \mu_{1} \geq 0$, and $\mu_{1} \leq 1$, but only two of these can be binding as we shall see. Hence, we define the Lagrangian $\mathcal{L}=\mathcal{L}\left(C_{1}, \mu_{1}\right)$ as

$$
\mathcal{L}=L_{1} U\left(C_{1} / L_{1}\right)+\nu_{1} S^{(1)}\left(K_{2}\right)-\nu_{2} S^{(2)}\left(M_{2}\right)+\kappa_{1} I_{1}+\kappa_{2}\left(1-\mu_{1}\right),
$$

and we find

$$
\frac{\partial \mathcal{L}}{\partial C_{1}}=U^{\prime}\left(C_{1} / L_{1}\right)-\left(\nu_{1} g_{1}+\kappa_{1}\right)
$$

and

$$
\frac{\partial \mathcal{L}}{\partial \mu_{1}}=\left(-\left(\nu_{1} g_{1}+\kappa_{1}\right) \psi_{1} \theta \mu_{1}^{\theta-1} d_{1}+\nu_{2} g_{2} \sigma_{1}\right) Y_{1}-\kappa_{2}
$$

where

$$
g_{1}=g_{1}\left(C_{1}, \mu_{1}\right)=\frac{\partial S^{(1)}\left(K_{2}\right)}{\partial K_{2}}, \quad g_{2}=g_{2}\left(\mu_{1}\right)=\frac{\partial S^{(2)}\left(M_{2}\right)}{\partial M_{2}} .
$$

This leads to the Kuhn-Tucker conditions:

$$
\begin{gathered}
\kappa_{1}=U^{\prime}\left(C_{1} / L_{1}\right)-\nu_{1} g_{1} \geq 0, \\
I_{1}=\left(1-\psi_{1} \mu_{1}^{\theta}\right) d_{1} Y_{1}-C_{1} \geq 0,
\end{gathered}
$$

and

$$
\begin{gathered}
\kappa_{2}=\left(-U^{\prime}\left(C_{1} / L_{1}\right) \psi_{1} \theta \mu_{1}^{\theta-1} d_{1}+\nu_{2} g_{2} \sigma_{1}\right) Y_{1} \geq 0 \\
\mu_{1} \leq 1,
\end{gathered}
$$

together with the slackness conditions $\kappa_{1} I_{1}=0$ and $\kappa_{2}\left(1-\mu_{1}\right)=0$.

Under the assumption that $I_{1}>0$ we have $\kappa_{1}=0$ and we distinguish between two cases, as follows. 
Case (1): $\kappa_{2}>0$. We have $\mu_{1}=1$ and $g_{2}=g_{2}(1)$, and we solve two equations in two unknowns:

$$
U^{\prime}\left(C_{1} / L_{1}\right)=\nu_{1} g_{1}, \quad g_{1}=g_{1}\left(C_{1}, 1\right)
$$

under the restrictions:

$$
\frac{C_{1}}{\left(1-\psi_{1}\right) Y_{1}} \leq d_{1}<\frac{\nu_{2} g_{2} \sigma_{1}}{\nu_{1} g_{1} \psi_{1} \theta}
$$

Case (2): $\kappa_{2}=0$. We solve four equations in four unknowns:

$$
\begin{gathered}
U^{\prime}\left(C_{1} / L_{1}\right)=\nu_{1} g_{1}, \quad \mu_{1}^{\theta-1} d_{1}=\frac{\nu_{2} g_{2} \sigma_{1}}{\nu_{1} g_{1} \psi_{1} \theta}, \\
g_{1}=g_{1}\left(C_{1}, \mu_{1}\right), \quad g_{2}=g_{2}\left(\mu_{1}\right),
\end{gathered}
$$

under the restrictions:

$$
C_{1} \leq\left(1-\psi_{1} \mu_{1}^{\theta}\right) d_{1} Y_{1}, \quad \mu_{1} \leq 1
$$

The following two points are worth noting. First, we see that the restrictions $\mu_{1} \geq 0$ and $C_{1} \geq 0$ are automatically satisfied, so that they do not need to be imposed. Second, we see that $U^{\prime}\left(C_{1} / L_{1}\right)=\nu_{1} g_{1}$ in both cases. This fact will be used in the proof of Proposition 6.1.

\section{B Proofs of the propositions}

Proof of Proposition 4.1: See Menger (1934, p. 468) in the context of St. Petersburg-type lotteries, and also Arrow (1974) and Gilboa (2009, pp. 108109). Menger (implicitly) assumes boundedness from below and demonstrates that boundedness from above should hold, and it is straightforward to generalize his result to an a priori unrestricted setting.

Proof of Proposition 4.2: Let $\alpha^{*}>0$. The EU maximizer is then more risk-averse in the sense of Arrow-Pratt than an agent with power (CRRA) utility of index $\alpha^{*}$. It follows from (4) that

$$
\frac{P^{\prime}\left(C_{1}^{*}\right)}{P\left(C_{1}^{*}\right)}=\frac{U^{\prime \prime}\left(C_{1}^{*}\right)}{U^{\prime}\left(C_{1}^{*}\right)}=-\operatorname{ARA}\left(C_{1}^{*}\right) .
$$


Since $\operatorname{ARA}(x)=\operatorname{RRA}(x) / x \geq \alpha^{*} / x$, we then have

$$
\begin{aligned}
\mathrm{E}(P) & =\frac{1}{1+\rho} \mathrm{E} \exp \left(-\int_{C_{1}^{*}}^{1} d \log P(x)\right)=\frac{1}{1+\rho} \operatorname{Eexp}\left(\int_{C_{1}^{*}}^{1} \operatorname{ARA}(x) d x\right) \\
& \geq \frac{1}{1+\rho} \int_{C_{1}^{*} \leq 1} \exp \left(\int_{C_{1}^{*}}^{1}\left(\alpha^{*} / x\right) d x\right) d F\left(\epsilon_{1}\right) \\
& =\frac{1}{1+\rho} \int_{C_{1}^{*} \leq 1}\left(C_{1}^{*}\right)^{-\alpha^{*}} d F\left(\epsilon_{1}\right) \geq \frac{B_{1}^{-\alpha^{*}}}{1+\rho} \int_{C_{1}^{*} \leq 1} e^{-\tau \alpha^{*} \epsilon_{1}} d F\left(\epsilon_{1}\right)=\infty,
\end{aligned}
$$

with

$$
B_{1}=\frac{e^{-\tau^{2} / 2} Y_{1}}{1+\xi H_{1}^{2}},
$$

using (5) and the fact that $\epsilon_{1}$ is heavy-tailed to the left. This proves part (a). Intuitively, if agent 1 is more risk-averse in the sense of Arrow-Pratt than agent 2 , and if it is optimal to postpone all consumption for agent 2, then this will also be optimal for agent 1 .

Next let $\alpha^{*}=0$ and $\beta^{*}<\infty$. The EU maximizer is then less risk-averse in the sense of Arrow-Pratt than an agent with exponential (CARA) utility of index $\beta^{*}$. Since $\alpha^{*}=0$, we have $0 \leq \operatorname{ARA}(x) \leq \beta^{*}$ and hence

$$
\begin{aligned}
\mathrm{E}(P)= & \int_{C_{1}^{*} \leq 1} P d F\left(\epsilon_{1}\right)+\int_{C_{1}^{*}>1} P d F\left(\epsilon_{1}\right) \\
\leq & \frac{1}{1+\rho} \int_{C_{1}^{*} \leq 1} \exp \left(\int_{C_{1}^{*}}^{1} \beta^{*} d x\right) d F\left(\epsilon_{1}\right) \\
& +\frac{1}{1+\rho} \int_{C_{1}^{*}>1} \exp \left(-\int_{1}^{C_{1}^{*}} \operatorname{ARA}(x) d x\right) d F\left(\epsilon_{1}\right) \\
\leq & \frac{e^{\beta^{*}} \operatorname{Pr}\left(C_{1}^{*} \leq 1\right)+\operatorname{Pr}\left(C_{1}^{*}>1\right)}{1+\rho}<\infty .
\end{aligned}
$$

Proof of Proposition 4.3: To prove the 'only if' part, we assume that $\int_{0}^{\gamma} \operatorname{ARA}(x) d x$ is infinite for every $\gamma>0$, and then show that there exist $(\mathcal{S}, \mathcal{A}, \mathbb{P})$ and $\epsilon_{1}$ defined on it such that $\mathrm{E}(P)=\infty$. We note that $\beta^{*}=\infty$. Define a function $g:(0,1] \rightarrow[1, \infty)$ by

$$
g(y)=\exp \left(\int_{y}^{1} \operatorname{ARA}(x) d x\right) .
$$

Then,

$$
\mathrm{E}(P) \geq \frac{1}{1+\rho} \int_{C_{1}^{*} \leq 1} g\left(\min \left(C_{1}^{*}, 1\right)\right) d F\left(\epsilon_{1}\right) .
$$


Recall from (5) that $C_{1}^{*} \leq B_{1} e^{\tau \epsilon_{1}}$, and let $\epsilon_{1}^{*}$ be such that $B_{1} e^{\tau \epsilon_{1}^{*}}=1$, so that $0<B_{1} e^{\tau \epsilon_{1}^{*}} \leq 1$ if and only if $\epsilon_{1} \leq \epsilon_{1}^{*}$. Define $u:(-\infty, \infty) \rightarrow[0, \infty)$ by

$$
u\left(\epsilon_{1}\right)= \begin{cases}g\left(B_{1} e^{\tau \epsilon_{1}}\right)-1 & \text { if } \epsilon_{1} \leq \epsilon_{1}^{*}, \\ 0 & \text { if } \epsilon_{1}>\epsilon_{1}^{*}\end{cases}
$$

Since $\operatorname{ARA}(1)>0, g$ is monotonically decreasing and we obtain

$$
\begin{gathered}
\int_{C_{1}^{*} \leq 1} g\left(\min \left(C_{1}^{*}, 1\right)\right) d F\left(\epsilon_{1}\right) \geq \int_{\epsilon_{1} \leq \epsilon_{1}^{*}} g\left(B_{1} e^{\tau \epsilon_{1}}\right) d F\left(\epsilon_{1}\right) \\
=\int_{\epsilon_{1} \leq \epsilon_{1}^{*}}(u+1) d F\left(\epsilon_{1}\right)=\mathrm{E}(u)+\operatorname{Pr}\left(\epsilon_{1} \leq \epsilon_{1}^{*}\right) .
\end{gathered}
$$

Strict monotonicity of $g$ implies its invertibility. Hence we can choose $u$ to be any non-negative random variable whose expectation does not exist (for example, the absolute value of a Cauchy distribution), and then define $\epsilon_{1}$ through $B_{1} e^{\tau \epsilon_{1}}=g^{-1}(u+1)$. With such a choice of $\epsilon_{1}$ we have $\mathrm{E}(P)=\infty$.

To prove the 'if'-part we assume that $\int_{0}^{\gamma} \operatorname{ARA}(x) d x$ is finite. This implies that $\int_{0}^{1} \operatorname{ARA}(x) d x$ is finite, so that

$$
\begin{aligned}
\mathrm{E}(P)= & \frac{1}{1+\rho} \int_{C_{1}^{*} \leq 1} \exp \left(\int_{C_{1}^{*}}^{1} \operatorname{ARA}(x) d x\right) d F\left(\epsilon_{1}\right) \\
& +\frac{1}{1+\rho} \int_{C_{1}^{*}>1} \exp \left(-\int_{1}^{C_{1}^{*}} \operatorname{ARA}(x) d x\right) d F\left(\epsilon_{1}\right) \\
\leq & \frac{\operatorname{Pr}\left(C_{1}^{*} \leq 1\right)}{1+\rho} \exp \left(\int_{0}^{1} \operatorname{ARA}(x) d x\right)+\frac{\operatorname{Pr}\left(C_{1}^{*}>1\right)}{1+\rho}<\infty
\end{aligned}
$$

using the fact that $\alpha^{*}=\operatorname{RRA}(0)=0$.

Proof of Proposition 6.1: We shall prove the proposition both for the linear scrap and the non-linear scrap case. In both cases the inequality constraints (1) are imposed. Since

$$
d_{1} Y_{1}=B_{1} e^{\tau \epsilon_{1}}, \quad B_{1}=\frac{e^{-\tau^{2} / 2} Y_{1}}{1+\xi H_{1}^{2}},
$$

we obtain

$$
\begin{gathered}
C_{1}^{*} \leq C_{1}^{*}+I_{1}^{*}=\left(1-\omega_{1}^{*}\right) d_{1} Y_{1} \leq B_{1} e^{\tau \epsilon_{1}} \\
I_{1}^{*} \leq C_{1}^{*}+I_{1}^{*} \leq B_{1} e^{\tau \epsilon_{1}} \\
(1-\delta) K_{1} \leq K_{2}^{*} \leq(1-\delta) K_{1}+B_{1} e^{\tau \epsilon_{1}}
\end{gathered}
$$


and

$$
M_{2}^{*} \leq(1-\phi) M_{1}+\sigma_{1} Y_{1} .
$$

We distinguish between three cases.

Linear scrap under normality. Linear scrap implies that $S^{(1)}\left(K_{2}\right)=K_{2}$ and $S^{(2)}\left(M_{2}\right)=M_{2}$. Since $\mathrm{E}\left(e^{\tau \epsilon_{1}}\right)$ exists under normality, it follows that $C_{1}^{*}, I_{1}^{*}$, $K_{2}^{*}$, and $M_{2}^{*}$ all have finite expectations, and therefore that $\mathrm{E}\left(W^{*}\right)$ exists if and only $\mathrm{E}\left(1 / C_{1}^{*}\right)$ exists. For notational convenience we do not distinguish between the random variable $\epsilon_{1}$ and its realization. With this slight abuse of notation, we write

$$
\begin{aligned}
\mathrm{E}\left(1 / C_{1}^{*}\right) & =\int_{-\infty}^{\infty}\left(1 / C_{1}^{*}\right) d F\left(\epsilon_{1}\right)=\int_{I_{1}^{*}=0}\left(1 / C_{1}^{*}\right) d F\left(\epsilon_{1}\right)+\int_{I_{1}^{*}>0}\left(1 / C_{1}^{*}\right) d F\left(\epsilon_{1}\right) \\
& =\left(1 / B_{1}\right) \int_{I_{1}^{*}=0} \frac{e^{-\tau \epsilon_{1}}}{1-\omega_{1}^{*}} d F\left(\epsilon_{1}\right)+\int_{I_{1}^{*}>0}\left(1 / C_{1}^{*}\right) d F\left(\epsilon_{1}\right) \\
& \leq \frac{1}{\left(1-\psi_{1}\right) B_{1}} \mathrm{E}\left(e^{-\tau \epsilon_{1}}\right)+\int_{I_{1}^{*}>0}\left(1 / C_{1}^{*}\right) d F\left(\epsilon_{1}\right) .
\end{aligned}
$$

Since $\mathrm{E}\left(e^{-\tau \epsilon_{1}}\right)$ is finite, it suffices to show that $\int_{I_{1}^{*}>0}\left(1 / C_{1}^{*}\right) d F\left(\epsilon_{1}\right)$ is finite. Now, it follows from Appendix A that, under the assumption that $I_{1}^{*}>0$, $U^{\prime}\left(C_{1}^{*} / L_{1}\right)=L_{1}^{2} / C_{1}^{* 2}=\nu_{1} g_{1}^{*}=\nu_{1}$, because $g_{1}^{*}=1$. Hence,

$$
\int_{I_{1}^{*}>0}\left(1 / C_{1}^{*}\right) d F\left(\epsilon_{1}\right)=\frac{\nu_{1}^{1 / 2}}{L_{1}} \operatorname{Pr}\left(I_{1}^{*}>0\right) \leq \frac{\nu_{1}^{1 / 2}}{L_{1}}<\infty .
$$

Nonlinear scrap under normality. Nonlinear scrap implies that

$$
S^{(1)}\left(K_{2}\right)=-\frac{K_{0}}{p}\left(\frac{K_{2}}{K_{0}}\right)^{-p}, \quad S^{(2)}\left(M_{2}\right)=\frac{M_{0}}{q}\left(\frac{M_{2}}{M_{0}}\right)^{q}
$$

where $p>0$ and $q>1$. Since

$$
\left(K_{2}^{*}\right)^{-p} \leq\left((1-\delta) K_{1}\right)^{-p}
$$

and

$$
\left(M_{2}^{*}\right)^{q} \leq\left((1-\phi) M_{1}+\sigma_{1} Y_{1}\right)^{q},
$$

we see that $\mathrm{E}\left(W^{*}\right)$ exists if and only $\mathrm{E}\left(1 / C_{1}^{*}\right)$ exists. As in the linear scrap case, it suffices to show that $\int_{I_{1}^{*}>0}\left(1 / C_{1}^{*}\right) d F\left(\epsilon_{1}\right)$ is finite. Since

$$
g_{1}=g_{1}\left(K_{2}\right)=\frac{\partial S^{(1)}\left(K_{2}\right)}{\partial K_{2}}=\left(\frac{K_{0}}{K_{2}}\right)^{p+1}
$$


it follows from Appendix A that, under the assumption that $I_{1}^{*}>0$,

$$
U^{\prime}\left(C_{1}^{*} / L_{1}\right)=L_{1}^{2} / C_{1}^{* 2}=\nu_{1} g_{1}^{*}=\nu_{1}\left(\frac{K_{0}}{K_{2}^{*}}\right)^{p+1} \leq \nu_{1}\left(\frac{K_{0}}{(1-\delta) K_{1}}\right)^{p+1},
$$

and hence that

$$
\int_{I_{1}^{*}>0}\left(1 / C_{1}^{*}\right) d F\left(\epsilon_{1}\right) \leq \frac{\nu_{1}^{1 / 2}}{L_{1}}\left(\frac{K_{0}}{(1-\delta) K_{1}}\right)^{(p+1) / 2}<\infty .
$$

Student distribution. From (9) we have $1 / C_{1}^{*} \geq e^{-\tau \epsilon_{1}} / B_{1}$. Under a Student distribution, the right-hand side has no finite expectation, and hence the left-hand side has no finite expectation either. In the non-linear scrap case, this is sufficient to prove the non-existence of $\mathrm{E}\left(W^{*}\right)$ because $S^{(1)}\left(K_{2}^{*}\right)$ and $S^{(2)}\left(M_{2}^{*}\right)$ are both bounded. In the linear scrap case, $M_{2}^{*}$ is bounded, but $K_{2}^{*}$ is not. Now, since

$$
C_{1}^{*} \leq B_{1} e^{\tau \epsilon_{1}}, \quad K_{2}^{*} \leq(1-\delta) K_{1}+B_{1} e^{\tau \epsilon_{1}},
$$

we obtain

$L_{1}\left(1-L_{1} / C_{1}^{*}\right)+\nu_{1} K_{2}^{*} \leq L_{1}-\left(L_{1}^{2} / B_{1}\right) e^{-\tau \epsilon_{1}}+\nu_{1}(1-\delta) K_{1}+\nu_{1} B_{1} e^{\tau \epsilon_{1}} \equiv G\left(\epsilon_{1}\right)$.

Since $G$ is monotonically increasing from $-\infty$ to $+\infty$, there exists a unique $\epsilon_{1}^{*}$ defined by $G\left(\epsilon_{1}^{*}\right)=0$. Hence, $G\left(\epsilon_{1}\right) \leq 0$ for all $\epsilon_{1} \leq \epsilon_{1}^{*}$ and

$$
\begin{aligned}
& \mathrm{E}\left|\left(L_{1}\left(1-L_{1} / C_{1}^{*}\right)+\nu_{1} K_{2}^{*}\right)\right| \geq \int_{\epsilon_{1} \leq \epsilon_{1}^{*}}\left|G\left(\epsilon_{1}\right)\right| d F\left(\epsilon_{1}\right) \\
& \quad \geq-L_{1}-\nu_{1}(1-\delta) K_{1}+\left(L_{1}^{2} / B_{1}\right) \int_{\epsilon_{1} \leq \epsilon_{1}^{*}} e^{-\tau \epsilon_{1}} d F\left(\epsilon_{1}\right)-\nu_{1} B_{1} e^{\tau \epsilon_{1}^{*}}=\infty .
\end{aligned}
$$

\section{References}

Ackerman, F., E.A. Stanton, and R. Bueno (2010). Fat tails, exponents, extreme uncertainty: Simulating catastrophe in DICE, Ecological Economics, 69, 1657-1665.

Arrow, K.J. (1974). The use of unbounded utility functions in expectedutility maximization: Response, Quarterly Journal of Economics, 88, $136-138$.

Barro, R.J. (2009). Rare disasters, asset prices, and welfare costs, American Economic Review, 99, 243-264. 
Broome, J.R. (1991). Weighing Goods, Basil Blackwell, Oxford, UK.

Fishburn, P.C. (1976). Unbounded utility functions in expected utility theory, Quarterly Journal of Economics, 90, 163-168.

Geweke, J. (2001). A note on some limitations of CRRA utility, Economics Letters, 71, 341-345.

Gilboa, I. (2009). Theory of Decision under Uncertainty, Cambridge University Press, Cambridge, UK.

Gollier, C. (2002). Time horizon and the discount rate, Journal of Economic Theory, 107, 463-473.

Gollier, C. (2008). Discounting with fat-tailed economic growth, Journal of Risk and Uncertainty, 37, 171-186.

Ikefuji, M., R.J.A. Laeven, J.R. Magnus, and C. Muris (2011a). Pareto utility, submitted for publication.

Ikefuji, M., R.J.A. Laeven, J.R. Magnus, and C. Muris (2011b). Weitzman meets Nordhaus: Background document, not for publication, http://www.janmagnus.nl/items/risk.pdf.

IPCC (2007). Solomon, S., D. Qin, M. Manning, Z. Chen, M. Marquis, K.B. Averyt, M. Tignor, and H.L. Miller (eds.), Contribution of Working Group I to the Fourth Assessment Report of the Intergovernmental Panel on Climate Change, Cambridge University Press, Cambridge, UK and New York, NY.

Keller, K., B.M. Bolker, and D.F. Bradford (2004). Uncertain climate thresholds and optimal economic growth, Journal of Environmental Economics and Management, 48, 723-741.

Kelly, D.L. and C.D. Kolstad (1999). Bayesian learning, growth, and pollution, Journal of Economic Dynamics and Control, 23, 491-518.

Leach, A.J. (2007). The climate change learning curve, Journal of Economic Dynamics and Control, 31, 1728-1752.

Manne, A.S. and R.G. Richels (1992). Buying Greenhouse Insurance: The Economic Costs of CO2 Emission Limits, MIT Press, Cambridge, MA.

Mastrandrea, M.D. and S.H. Schneider (2004). Probabilistic integrated assessment of 'dangerous' climate change, Science, 304, 571-575. 
Menger, K. (1934). Das Unsicherheitsmoment in der Wertlehre, Zeitschrift für Nationalökonomie, 5, 459-485.

Moscadelli, M. (2004). The modelling of operational risk: Experience with the analysis of the data collected by the Basel Committee, Technical Report 517, Banca d'Italia.

Nordhaus, W.D. (1994). Managing the Global Commons: The Economics of Climate Change, MIT Press, Cambridge, MA.

Nordhaus W.D. (2008). A Question of Balance: Weighing the Options on Global Warming Policies, The Challenge of Global Warming: Economic Models and Environmental Policy, Yale University Press, New Haven, CT.

Nordhaus, W.D. and Z. Yang (1996). A regional dynamic general-equilibrium model of alternative climate-change strategies, American Economic Review, 86, 741-765.

Roughgarden, T. and S.H. Schneider (1999). Climate change policy: Quantifying uncertainties for damages and optimal carbon taxes, Energy Policy, 27, 415-429.

Ryan, T.M. (1974). The use of unbounded utility functions in expectedutility maximization: Comment, Quarterly Journal of Economics, 88, $133-135$.

Savage, L.J. (1954). The Foundations of Statistics, Wiley, New York, NY.

Sims, C.A. (2001). Pitfalls of a minimax approach to model uncertainty, American Economic Review, 91, 51-54.

Stern, N. (2007). The Economics of Climate Change: The Stern Review, Cambridge University Press, Cambridge, UK.

Ursúa, J.F. (2010). Long-run volatility, Harvard University.

Von Neumann, J. and O. Morgenstern $(1944,1947,1953)$. Theory of Games and Economic Behavior, Princeton University Press, Princeton, NJ.

Weitzman, M.L. (2009). On modeling and interpreting the economics of catastrophic climate change, Review of Economics and Statistics, 91, $1-19$. 